\title{
THE ORBIT AND PULSATION PERIODS OF THE $\gamma$ DORADUS VARIABLE HR 6844 (V2502 OPHIUCHI)
}

\author{
Francis C. FEKEL ${ }^{1,2}$ AND GREgORY W. HENRY ${ }^{2}$ \\ Center of Excellence in Information Systems, Tennessee State University, 330 10th Avenue North, Nashville, TN 37203; \\ fekel@evans.tsuniv.edu,henry@schwab.tsuniv.edu \\ Received 2002 November 12; accepted 2002 December 16
}

\begin{abstract}
We obtained spectroscopic and photometric observations of the $\gamma$ Doradus variable HR $6844(=\mathrm{V} 2502$ Ophiuchi). Radial velocities show that this star is a single-lined binary with a period of 4.4852 days. The primary is an F1 V star, while the secondary is likely an M dwarf. Velocity residuals to a circular orbit have a period of 1.3071 days and an amplitude of $\sim 3 \mathrm{~km} \mathrm{~s}^{-1}$. Three periods of light variation were detected, 1.30702, 1.4350 , and 0.62286 days. The first period is essentially identical to that found in the radial velocities and has the largest amplitude, a peak-to-peak value of $0.067 \mathrm{mag}$ in $B$. The photometric check star, $73 \mathrm{Oph}(=\mathrm{HR}$ 6795), has light variations with a period of 0.61439 in $B$. Although the star is a close visual binary, the light variations are ascribed to the primary, making it most likely a newly discovered $\gamma$ Doradus variable.
\end{abstract}

Key words: binaries: spectroscopic — stars: fundamental parameters — stars: variables: other

On-line material: machine-readable table

\section{INTRODUCTION}

The Hipparcos mission team discovered HR 6844 (HD $167858=\mathrm{V} 2502 \quad \mathrm{Oph} ; \quad \alpha=18^{\mathrm{h}} 17^{\mathrm{m}} 04 \mathrm{~s} .8, \quad \delta=01^{\circ} 00^{\prime} 20^{\prime \prime} \cdot 6$ [J2000.0], $V=6.62 \mathrm{mag}, \mathrm{F} 1 \mathrm{~V}$ ) to be variable with an amplitude of $\sim 0.1 \mathrm{mag}$ and a period of 1.307 days (ESA 1997). Reanalyzing the photometry from the Hipparcos database, Aerts, Eyer, \& Kestens (1998) and Paunzen \& Maitzen (1998) identified HR 6844 as a $\gamma$ Doradus candidate, while Handler (1999) included the star in his list of prime candidates. From additional ground-based photometry Handler $\&$ Shobbrook (2002) confirmed it as a $\gamma$ Doradus variable.

On the basis of the properties of 13 stars, Kaye et al. (1999a) defined the class of $\gamma$ Doradus variables. Objects identified so far straddle the red edge of the $\delta$ Scuti instability strip and are dwarfs or subgiants with late A or early F spectral classes. The periods of the stars range from about 0.3 to 3.0 days, with the low end of this range slightly overlapping the periods of the most luminous $\delta$ Scuti stars. Like the $\delta$ Scuti variables, most $\gamma$ Doradus stars have multiple photometric periods. The photometric amplitudes are modest and range up to $\sim 0.1 \mathrm{mag}$ in Johnson $V$. Line profile changes, resulting in radial velocity variations of $1-4 \mathrm{~km}$ $\mathrm{s}^{-1}$, have been documented in several stars (e.g., Krisciunas et al. 1995; Balona et al. 1996; Aerts \& Kaye 2001). The light and line profile variations most likely result from nonradial, $g$-mode pulsations of high order $(n)$ and low spherical degree (l) (Kaye et al. 1999a). Guzik et al. (2000) developed the first models of a driving mechanism for these gravitymode pulsations. To date, only 30 stars have been confirmed as $\gamma$ Doradus variables (Henry \& Fekel 2002).

HR 6844 is one of a small number of very slowly rotating, early F-type stars. Danziger \& Faber (1972) found $v \sin i \leq 10 \mathrm{~km} \mathrm{~s}^{-1}$, while more recent values are 13 (Abt \& Morrell 1995), 8.0 (Fekel 1997), and $8.1 \mathrm{~km} \mathrm{~s}^{-1}$ (Strassmeier

\footnotetext{
${ }^{1}$ Visiting Astronomer, Kitt Peak National Observatory, National Optical Astronomy Observatory, which is operated by the Association of Universities for Research in Astronomy, Inc., under cooperative agreement with the National Science Foundation.

${ }^{2}$ Senior Research Associate, Department of Physics and Astronomy, Vanderbilt University, Nashville, TN 37235.
}

et al. 2000). Cowley \& Fraquelli (1974) classified it as F2 V, in good agreement with the spectral type of F1 V determined more recently by both Gray \& Garrison (1989) and Abt \& Morrell (1995). From a preliminary analysis of our spectroscopic data, Fekel, Warner, \& Kaye (2003) announced that HR 6844 is a single-lined spectroscopic binary with a period of 4.485 days but also has additional velocity variability that is detectable on a timescale of hours. Because of these additional short-period velocity variations, we also obtained photometric observations of HR 6844. Here we present an extensive analysis of both sets of data.

\section{SPECTROSCOPY}

\subsection{Observations}

From 1993 April to 2002 April, we obtained 44 high-resolution, red-wavelength spectrograms with the Kitt Peak National Observatory (KPNO) coudé feed telescope, coudé spectrograph, and a TI CCD detector. Uusally, a single observation was obtained per night, but in 2001 September we made multiple observations on three consecutive nights. All but one of the KPNO spectrograms are centered in the red at $6430 \AA$, cover a wavelength range of about $80 \AA$, and have a resolution of $0.21 \AA$. A lone spectrogram was centered at $6700 \AA$. The spectra have typical signal-to-noise ratios of $150-200$.

Radial velocities (Table 1) were determined in the 6385$6444 \AA$ region with the IRAF ${ }^{3}$ cross-correlation program FXCOR (Fitzpatrick 1993). The IAU radial velocity standards HR 5694 and HR 7560 were used as reference stars. Their velocities of 54.4 and $0.0 \mathrm{~km} \mathrm{~s}^{-1}$, respectively, were adopted from Scarfe, Batten, \& Fletcher (1990).

\subsection{Orbit of HR $6844(=H D 167858=$ V2502 Ophiuchi $)$}

An initial spectroscopic period of 4.485 days was determined by fitting a sine curve to the velocities for trial periods between 0.1 and 10 days with a step size of 0.001

${ }^{3}$ IRAF is distributed by the National Optical Astronomy Observatory. 
TABLE 1

RAdial Velocities of HR 6844

\begin{tabular}{|c|c|c|c|}
\hline $\begin{array}{c}\text { HJD } \\
(-2,400,000)\end{array}$ & Phase & $\begin{array}{l}\text { Velocity } \\
\left(\mathrm{km} \mathrm{s}^{-1}\right)\end{array}$ & $\begin{array}{c}O-C \\
\left(\mathrm{~km} \mathrm{~s}^{-1}\right)\end{array}$ \\
\hline $49,106.000 .$. & 0.511 & -36.3 & -1.7 \\
\hline $49,250.617 \ldots \ldots \ldots \ldots \ldots \ldots$ & 0.755 & -27.1 & 1.1 \\
\hline $49,837.978 \ldots \ldots \ldots \ldots \ldots \ldots$ & 0.711 & -29.6 & 0.3 \\
\hline $49,968.676 \ldots \ldots \ldots \ldots \ldots \ldots$ & 0.851 & -25.6 & -0.9 \\
\hline $49,971.694 \ldots \ldots \ldots \ldots \ldots \ldots$ & 0.524 & -35.5 & -1.0 \\
\hline $50,575.999 \ldots \ldots \ldots \ldots \ldots \ldots$ & 0.257 & -28.1 & 0.6 \\
\hline $50,630.859 \ldots$ & 0.489 & -34.0 & 0.6 \\
\hline $50,631.798 \ldots \ldots \ldots \ldots \ldots \ldots$ & 0.698 & -33.5 & -3.1 \\
\hline $50,633.800 \ldots \ldots \ldots \ldots \ldots \ldots$ & 0.144 & $-23.4^{\mathrm{a}}$ & 1.2 \\
\hline $50,634.878 \ldots$. & 0.385 & -32.3 & 0.7 \\
\hline $50,718.617 \ldots \ldots \ldots \ldots \ldots \ldots$ & 0.055 & -20.9 & 1.7 \\
\hline $50,757.546 \ldots \ldots \ldots \ldots \ldots \ldots$ & 0.734 & -27.9 & 1.1 \\
\hline $50,926.988 \ldots \ldots$ & 0.513 & -34.2 & 0.4 \\
\hline $50,927.996 \ldots \ldots \ldots \ldots \ldots \ldots$ & 0.737 & -28.4 & 0.5 \\
\hline $50,928.983 \ldots \ldots \ldots \ldots \ldots \ldots$ & 0.957 & -21.2 & 1.2 \\
\hline $50,931.962 \ldots$ & 0.622 & -33.0 & -0.1 \\
\hline $51,003.704 \ldots \ldots$ & 0.617 & -33.6 & -0.6 \\
\hline $51,004.805 \ldots \ldots$ & 0.862 & -24.2 & 0.2 \\
\hline $51,088.605 \ldots \ldots \ldots \ldots \ldots \ldots$ & 0.546 & -34.8 & -0.5 \\
\hline $51,089.655 \ldots \ldots \ldots \ldots \ldots \ldots$ & 0.780 & -27.0 & 0.2 \\
\hline $51,090.584 \ldots \ldots \ldots \ldots \ldots \ldots$ & 0.987 & -22.7 & -0.5 \\
\hline $51,091.623 \ldots \ldots \ldots \ldots \ldots \ldots$ & 0.219 & -28.9 & -1.7 \\
\hline $51,092.573 \ldots \ldots \ldots \ldots \ldots \ldots$ & 0.431 & -35.0 & -1.0 \\
\hline $51,093.610 \ldots \ldots \ldots \ldots \ldots \ldots$ & 0.662 & -28.3 & 3.3 \\
\hline $51,094.599 \ldots \ldots$ & 0.883 & -25.2 & -1.4 \\
\hline $51,306.922 \ldots \ldots$ & 0.221 & -27.1 & 0.2 \\
\hline $51,350.797 \ldots \ldots \ldots \ldots \ldots$ & 0.004 & -22.4 & -0.2 \\
\hline $51,352.868 \ldots \ldots \ldots \ldots \ldots \ldots$ & 0.465 & -35.5 & -1.1 \\
\hline $51,471.610 \ldots \ldots \ldots \ldots \ldots \ldots$ & 0.940 & -23.4 & -0.8 \\
\hline $51,472.562 \ldots \ldots \ldots \ldots \ldots \ldots$ & 0.152 & -23.9 & 0.9 \\
\hline $51,732.796 \ldots \ldots \ldots \ldots \ldots \ldots$ & 0.173 & -25.2 & 0.3 \\
\hline $51,807.613 \ldots \ldots \ldots \ldots \ldots \ldots$ & 0.854 & -24.9 & -0.3 \\
\hline $52,179.592 \ldots \ldots \ldots \ldots \ldots \ldots$ & 0.789 & -25.2 & 1.7 \\
\hline $52,179.721 \ldots \ldots \ldots \ldots \ldots \ldots$ & 0.818 & -23.8 & 2.0 \\
\hline $52,180.583 \ldots \ldots \ldots \ldots \ldots \ldots$ & 0.010 & -24.7 & -2.5 \\
\hline $52,180.739 \ldots \ldots \ldots \ldots \ldots \ldots$ & 0.045 & -22.7 & -0.3 \\
\hline $52,181.582 \ldots \ldots \ldots \ldots \ldots \ldots$ & 0.233 & -27.7 & 0.0 \\
\hline $52,181.648 \ldots \ldots \ldots \ldots \ldots \ldots$ & 0.247 & -28.8 & -0.5 \\
\hline $52,181.734 \ldots \ldots \ldots \ldots \ldots \ldots$ & 0.267 & -30.1 & -1.1 \\
\hline $52,182.711 \ldots \ldots \ldots \ldots \ldots \ldots$ & 0.484 & -35.4 & -0.8 \\
\hline $52,390.928 \ldots \ldots \ldots \ldots \ldots \ldots$ & 0.908 & -26.0 & -2.8 \\
\hline $52,392.003 \ldots \ldots \ldots \ldots \ldots \ldots$ & 0.147 & -23.3 & 1.4 \\
\hline $52,392.925 \ldots \ldots \ldots \ldots \ldots \ldots$ & 0.353 & -30.1 & 2.0 \\
\hline $52,393.011 \ldots \ldots \ldots \ldots \ldots \ldots$ & 0.372 & -31.5 & 1.2 \\
\hline
\end{tabular}

${ }^{\text {a }}$ Lithium region, central wavelength $6700 \AA$.

days. For each period the sum of the residuals squared was computed, and the period with the smallest value of that sum was identified as the preliminary value of the orbital period. With this period adopted, initial orbital elements were computed with BISP (Wolfe, Horak, \& Storer 1967), a computer program that implements a slightly modified version of the Wilsing-Russell method. The orbit was then refined with SB1 (Barker, Evans, \& Laing 1967), a program that uses differential corrections. The eccentricity of this orbit is $0.090 \pm 0.042$, and so a circular orbit was also computed. To determine which orbit is to be preferred, we first used the two tests of Lucy \& Sweeney (1971), but those tests produced conflicting results. The eccentric-orbit solution resulted in a standard error of $1.12 \mathrm{~km} \mathrm{~s}^{-1}$ for an observation of unit
TABLE 2

ORBitAl ELEMENTS OF HR 6844

\begin{tabular}{|c|c|}
\hline Parameter & Value \\
\hline$P($ days $) \ldots \ldots$ & $4.48518 \pm 0.00019$ \\
\hline$T_{0}(\mathrm{HJD}) \ldots$ & $2,450,525.508 \pm 0.046$ \\
\hline 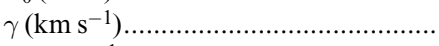 & $-28.40 \pm 0.22$ \\
\hline 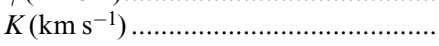 & $6.20 \pm 0.30$ \\
\hline 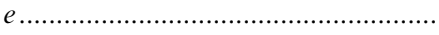 & 0.0 adopted \\
\hline 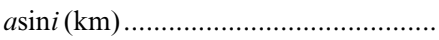 & $0.382 \pm 0.019 \times 10^{6}$ \\
\hline$f(m)\left(M_{\odot}\right) \ldots \ldots \ldots \ldots$ & $0.000111 \pm 0.000016$ \\
\hline $\begin{array}{l}\text { Standard error of an observation } \\
\text { of unit weight }\end{array}$ & $1.37 \mathrm{~km} \mathrm{~s}^{-1}$ \\
\hline
\end{tabular}

weight. Such a value is much larger than the values of $0.2-0.5 \mathrm{~km} \mathrm{~s}^{-1}$ (e.g., Fekel et al. 2002) that are usually found for the orbits of late-type stars, determined with the same instrument and detector combination.

As discussed in $\S 2.3$, an additional periodicity of 1.307 days has been found in the radial velocity data. Although this velocity variation results from pulsation, as shown in $\S 3.3$, D. Barlow (2002, private communication) kindly computed an orbital solution that solved for both periodicities simultaneously. The resulting periods are essentially identical to those found by analyzing the data sequentially, obtaining the orbital motion first and then determining the pulsation period from the velocity residuals of a circular orbit. The 1.307 day velocity variation was then subtracted from the observed velocities. The resulting orbital solution had an eccentricity of $0.038 \pm 0.035$, and so, according to the tests of Lucy \& Sweeney (1971), the orbit should be considered circular. Additional justification for a circular orbit is discussed in $\S 4$.

Elements of the adopted orbit are listed in Table 2. Since a time of periastron passage is undefined in a circular orbit, as recommended by Batten, Fletcher, \& MacCarthy (1989), $T_{0}$, a time of maximum radial velocity, is listed instead. The orbital phases and velocity residuals of the orbital solution are given in Table 1. The observed velocities and the computed velocity curve are compared in Figure 1, where zero phase is a time of maximum velocity.

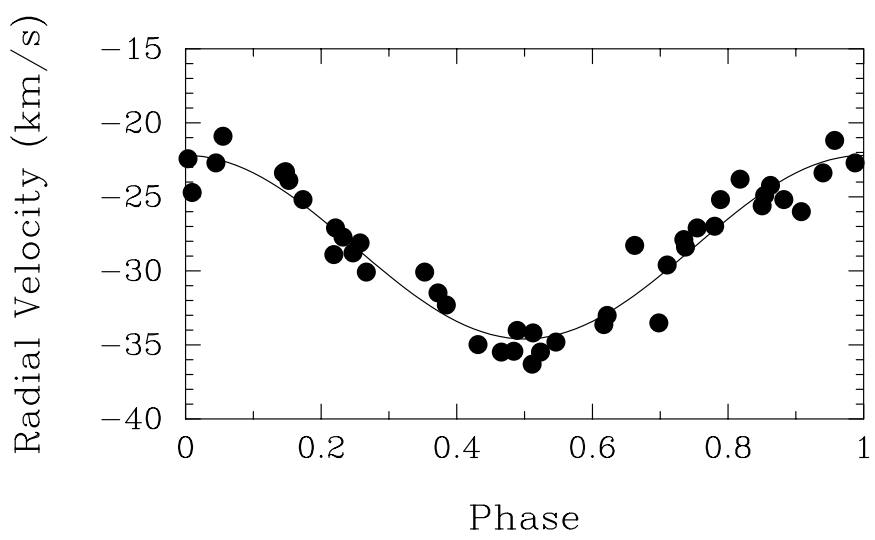

FIG. 1.-Computed radial velocity curve of HR 6844 compared with the observations. Zero phase is a time of maximum velocity. The plot shows the relatively large scatter of $1.37 \mathrm{~km} \mathrm{~s}^{-1}$ in the velocities around the calculated curve, as well as systematic trends in velocity at some phases. 


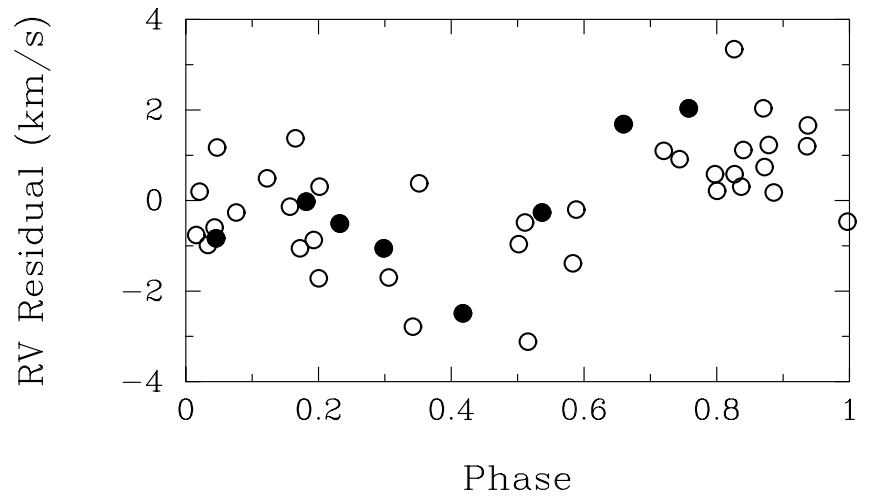

FIG. 2.-Radial velocity residuals from the circular orbit fit plotted with a period of 1.3071 days. Filled circles are the observations obtained on four consecutive nights in 2001 September. Zero phase is HJD 2,449,105.738.

\subsection{Velocity Residuals}

As noted above, the large velocity residuals to the circular orbit indicate that HR 6844 has additional velocity variations. We used the period finding program to search the velocity residuals of the orbital fit and found a period of 1.3071 days, essentially identical to the light variability period found in both our photometric data ( $\S 3.3$ below) and those from Hipparcos (ESA 1997). Figure 2 shows the velocity residuals plotted modulo the 1.3071 day period. Observations taken on four consecutive nights in 2001 September are highlighted. The velocity changes have an amplitude of $\sim 3 \mathrm{~km}^{-1}$, which is nearly one-fourth the amplitude of the orbital variation. The large scatter of the velocities suggests that additional periods are present in the data, but a search for them proved negative.

\section{PHOTOMETRY}

\subsection{Observations}

The photometric observations were acquired between 1999 May and 2001 June with the T3 0.4 m automatic photoelectric telescope (APT) at Fairborn Observatory. The 0.4 m APT uses a temperature-stabilized EMI 9924B photomultiplier tube to acquire data successively through Johnson $B$ and $V$ filters. The measurements of our program star HR 6844 were made in the following sequence, termed a group observation: $K, S, C, V, C, V, C, V, C, S, K$, in which $K$ is a check star, $C$ is the comparison star, $V$ is the program star, and $S$ is a sky reading. The comparison star was HD $166073(V=6.99, B-V=0.45, \mathrm{~F} 7 \mathrm{IV})$; the check star was 73 Oph $(V=5.71, B-V=0.36, \mathrm{~F} 2 \mathrm{~V})$. Typically, one group observation was acquired each clear night, but five nights were devoted to monitoring the group for several hours and resulted in 50-60 group observations per night. Three $V-C$ and two $K-C$ differential magnitudes were formed from each sequence and averaged together to create group means. Group mean differential magnitudes with internal standard deviations greater than $0.01 \mathrm{mag}$ were rejected to filter the observations taken under nonphotometric conditions. The surviving group means were corrected for differential extinction with nightly extinction coefficients, transformed to the Johnson system with yearly mean transformation coefficients, and treated as single observations thereafter. The external precision of our differ-
TABLE 3

Photometric ObServations of HR 6844 (Var) AND 73 Oph (CHK)

\begin{tabular}{ccccc}
\hline $\begin{array}{c}\text { HJD } \\
(-2,400,000)\end{array}$ & $\begin{array}{c}\mathrm{Var}_{B} \\
(\mathrm{mag})\end{array}$ & $\begin{array}{c}\mathrm{Var}_{V} \\
(\mathrm{mag})\end{array}$ & $\begin{array}{c}\mathrm{Chk}_{B} \\
(\mathrm{mag})\end{array}$ & $\begin{array}{r}\mathrm{Chk}_{V} \\
(\mathrm{mag})\end{array}$ \\
\hline $51,326.9259 \ldots \ldots \ldots$ & -0.470 & -0.345 & -1.361 & -1.292 \\
$51,327.7720 \ldots \ldots \ldots$. & -0.469 & -0.340 & 99.999 & 99.999 \\
$51,327.9232 \ldots \ldots \ldots$. & -0.458 & -0.325 & -1.344 & -1.265 \\
$51,329.8419 \ldots \ldots \ldots$. & -0.538 & -0.395 & -1.355 & -1.283 \\
$51,330.9344 \ldots \ldots \ldots$. & -0.481 & -0.347 & -1.347 & -1.269 \\
\hline
\end{tabular}

Notes.-Table 3 is presented in its entirety in the electronic edition of the Astronomical Journal. A portion is shown here for guidance regarding its form and content.

ential magnitudes, defined as the standard deviation of a single differential magnitude from the seasonal mean of the differential magnitudes and determined from observations of pairs of constant stars, is $\sim 0.005 \mathrm{mag}$. The individual photometric observations are given in Table 3 and are also available on the Tennessee State University Automated Astronomy Group Web site. ${ }^{4}$ The $B$ observations are plotted versus Julian date in Figure 3, while the data from the five individual monitoring nights are shown separately in Figure 4.

\subsection{Period Analysis}

We applied the method of Vanicek (1971), as described in Henry et al. (2001), to search for periodicities in the photometric data. We analyzed the program star minus comparison star $(V-C)$ differential magnitudes in $B$ and $V$ over the frequency range $0.01-30.0 \mathrm{day}^{-1}$, which corresponds to the period range 0.033-100 days. Our initial period searches were performed on our full data sets, including the five monitoring nights. However, we found that cycle-to-cycle changes in the level of maximum brightness (see $\S 3.3$ below), combined with the uneven phase coverage resulting from inclusion of the five monitoring nights, gave unsatisfactory results. Therefore, to produce a more uniformly sampled light curve, we removed the monitoring data, except for the first, middle, and last observations from those five nights, and repeated

${ }^{4}$ See http://schwab.tsuniv.edu/papers/aj/hr6844/hr6844.html.

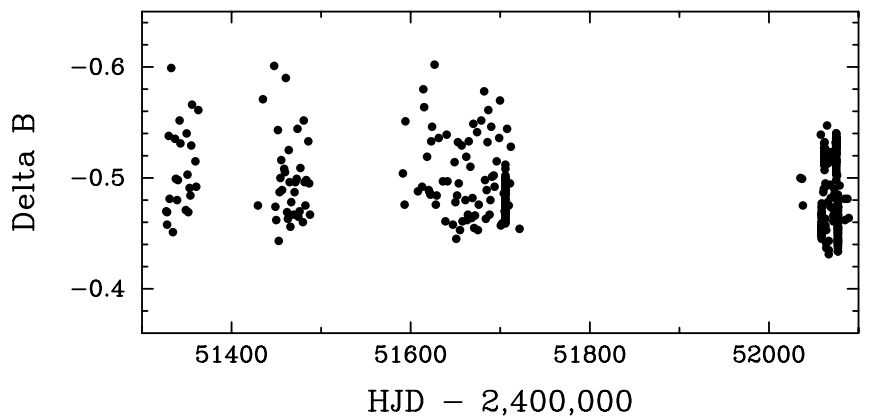

FIG. 3.-Complete set of HR 6844 photometric observations in Johnson $B$ acquired with the T3 automatic telescope between 1999 May and 2001 June. On most nights, the telescope made one observation of HR 6844, but 50 or more observations were obtained over several hours on each of five nights chosen to monitor the star more extensively. 


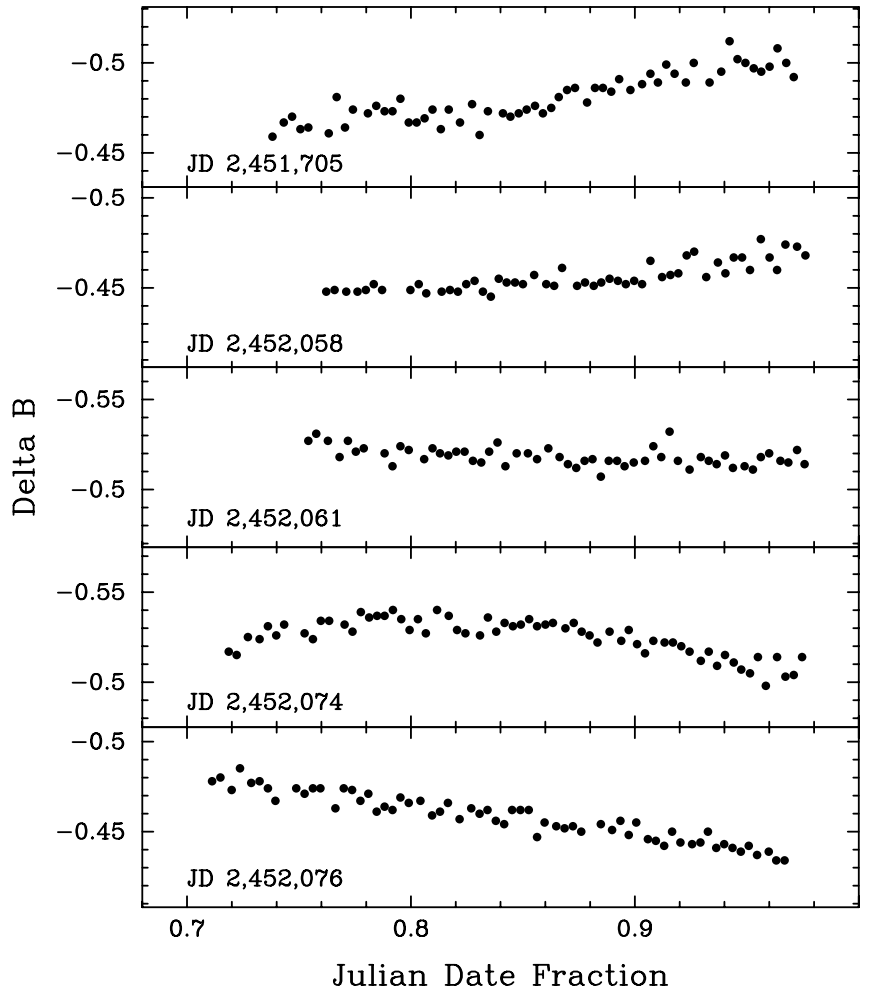

FIG. 4.-Individual Johnson $B$ observations from the five monitoring nights. These observations suggest a photometric period of approximately 0.6-0.7 days, but the level changes seen from day to day indicate at least one additional, longer period. Only the first, middle, and last observations from each panel were included in our period search, as described in the text.

the analysis. The results are listed in Table 4. The number of observations in column (4) refers to the modified data sets. The frequencies and periods given in columns (5) and (6), respectively, are included only if they were detected independently in both the $B$ and $V$ data. The peak-to-peak amplitudes reported in column (7) are determined for each frequency without prewhitening for the other frequencies. Times of minimum light for each frequency are given in column (8); the times of minimum in the two passbands agree within their uncertainites, so there are no detectable phase shifts in our two color photometry. Results for HR 6844 are discussed in $\S 3.3$ below.

We also analyzed the check star minus comparison star $(K-C)$ differential magnitudes in the same way to search for the possibility of photometric variations in our check and comparison stars. The results imply that our comparison star has no detectable periodicity, but our check star, 73 Oph, is a new $\gamma$ Doradus variable. Therefore, we include it in Table 4 and discuss these results in $\S 6$.

\subsection{Results for HR 6844}

The least-squares spectra for our $B$ data set (modified as described in $\S 3.2$ above) are shown in Figure 5. Although the analysis was done over the frequency range of 0.01-30.0 day $^{-1}$, the least-squares spectra are plotted only to 5 day $^{-1}$, since no variability was seen at higher frequencies. The four panels of Figure 5 illustrate the result of successively fixing the detected frequencies in turn and searching for additional ones. The three frequencies detected in this process are $0.76510,0.69688$, and $1.60551 \mathrm{day}^{-1}$; these correspond to periods of $1.30702,1.43497$, and 0.62286 days with peak-topeak amplitudes of $67.4,39.3$, and $33.3 \mathrm{mmag}$, respectively. The third frequency has an alias at 0.605 day $^{-1}$ (1.65 days) that is very nearly as strong, but the 0.62 day period is consistent with the data from the five monitoring nights in Figure 4. As reported in Table 4, the same three periods were detected in the modified $V$ data set. The amplitudes in $B$ average 1.39 times larger than the amplitudes in $V$. The 1.307 day variation, which has the largest amplitude in both photometric passbands, matches the period detected in the radial velocity residuals to the orbital solution, as discussed in $\S 2.3$ above, indicating that the effects of pulsation are evident in the radial velocities.

The 1.307 day period was detected previously in the Hipparcos photometry by Aerts et al. (1998) and Handler (1999), so we confirm that result in both our photometry and spectroscopy. In addition, Aerts et al. (1998) reported a second period at 0.9165 days, while Handler (1999) gave an average value of 0.85 days for several possible additional periods. We found three periods in our APT data but see no evidence for the second period reported by Aerts et al. (1998) or for any period around Handler's mean value of 0.85 days. These conflicting results are not totally

TABLE 4

Results from Photometric ANalysis

\begin{tabular}{|c|c|c|c|c|c|c|c|}
\hline $\begin{array}{c}\text { Star } \\
\text { (1) }\end{array}$ & $\begin{array}{l}\text { Photometric } \\
\text { Band } \\
\text { (2) }\end{array}$ & $\begin{array}{c}\text { Date Range } \\
(\mathrm{HJD}-2,450,000) \\
(3)\end{array}$ & $\begin{array}{c}N_{\mathrm{obs}}{ }^{\mathrm{a}} \\
\text { (4) }\end{array}$ & $\begin{array}{l}\text { Frequency } \\
\left(\text { day }^{-1}\right) \\
(5)\end{array}$ & $\begin{array}{l}\text { Period } \\
\text { (days) } \\
(6)\end{array}$ & $\begin{array}{l}\text { Peak-to-Peak } \\
\text { Amplitude } \\
\text { (mmag) } \\
\text { (7) }\end{array}$ & $\begin{array}{c}T_{\min } \\
(\mathrm{HJD}-2,450,000) \\
(8)\end{array}$ \\
\hline \multirow[t]{6}{*}{ HR $6844 \ldots \ldots}$. & $B$ & $1,326.9259-2,088.8987$ & 186 & $0.76510 \pm 0.00006$ & $1.30702 \pm 0.00009$ & $67.4 \pm 5.6$ & $1700.645 \pm 0.018$ \\
\hline & & & & $0.69688 \pm 0.00006$ & $1.43497 \pm 0.00012$ & $39.3 \pm 7.1$ & $1700.893 \pm 0.041$ \\
\hline & & & & $1.60551^{b} \pm 0.00005$ & $0.62286^{\mathrm{b}} \pm 0.00002$ & $33.3 \pm 7.2$ & $1700.123 \pm 0.022$ \\
\hline & $V$ & $1,326.9259-2,088.8987$ & 183 & $0.76508 \pm 0.00006$ & $1.30705 \pm 0.00010$ & $47.8 \pm 4.5$ & $1700.642 \pm 0.020$ \\
\hline & & & & $0.69688 \pm 0.00006$ & $1.43497 \pm 0.00012$ & $29.0 \pm 5.4$ & $1700.896 \pm 0.043$ \\
\hline & & & & $1.60554^{b} \pm 0.00005$ & $0.62284^{b} \pm 0.00002$ & $23.9 \pm 5.4$ & $1700.120 \pm 0.023$ \\
\hline \multirow[t]{2}{*}{$73 \mathrm{Oph} \ldots \ldots \ldots . .}$. & $B$ & $1,326.9259-2,088.8987$ & 171 & $1.62762 \pm 0.00005$ & $0.61439 \pm 0.00002$ & $8.6^{c} \pm 1.3$ & $1700.184 \pm 0.015$ \\
\hline & $V$ & $1,326.9259-2,088.8987$ & 175 & $1.63029 \pm 0.00005$ & $0.61339 \pm 0.00005$ & $6.2^{\mathrm{c}} \pm 1.4$ & $1700.209 \pm 0.022$ \\
\hline
\end{tabular}

a The individual observations are given in Table 3 .

b A probable alias at $0.605 \mathrm{day}^{-1}$, corresponding to a period of 1.65 days, is nearly as strong in the power spectrum but seems inconsistent with the data from the five monitoring nights in Fig. 4.

${ }^{\mathrm{c}}$ The photometric amplitude has not been corrected for dilution by the secondary component of the visual binary. 


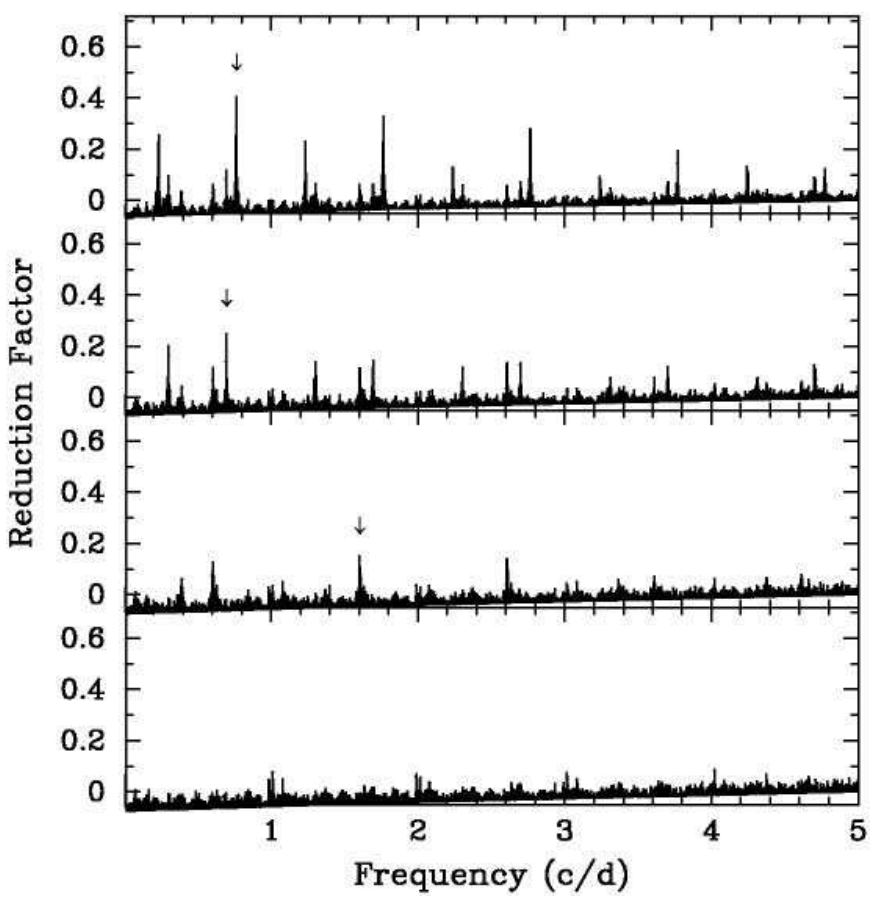

FIG. 5.- Least-squares spectra of the HR 6844 Johnson $B$ data, showing the results of progressively fixing the three detected frequencies. The arrows indicate the three frequencies (top to bottom): $0.76510,0.69688$, and $1.60551 \mathrm{day}^{-1}$. All three frequencies were confirmed in the Johnson $V$ data set.

surprising, since both Aerts et al. (1998) and Handler (1999) commented on the possibility of spurious periods due to the sampling of the Hipparcos photometry and, as a result, called for follow-up, ground-based observations.

The modified $B$ data set is phased with each of the three frequencies and times of minimum from Table 4 and plotted in Figure 6. For each panel, the data have been prewhitened to remove the other two frequencies. The light variations at all three frequencies are approximately sinusoidal. How-

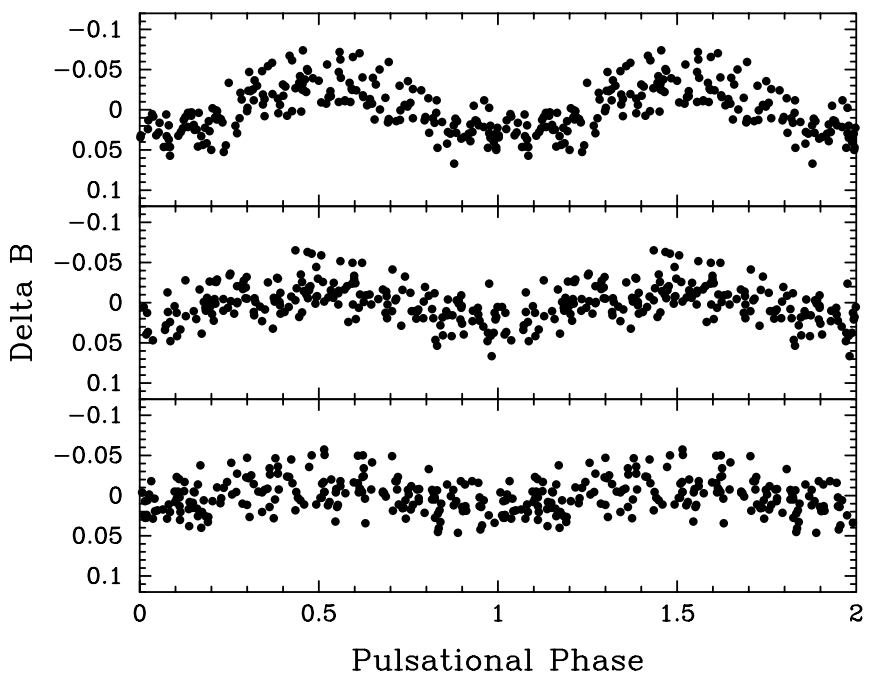

FIG. 6.-Johnson $B$ photometric data for HR 6844, phased with the three frequencies and times of minimum from Table 4. (Top to bottom): Frequencies are $0.76510,0.69688$, and $1.60551 \mathrm{day}^{-1}$. For each panel, the data set has been prewhitened to remove the other two known frequencies. ever, the top panel for the 1.307 day period clearly shows excess scatter around maximum brightness, while little or no such excess scatter is seen for the other two periods. This cycle-to-cycle variation in the level of maximum brightness, similar to the Blazhko effect observed in some RR Lyrae variables (Szeidl 1976), is also seen in several other $\gamma$ Doradus variables [e.g., 9 Aurigae $=$ V398 Aur (Zerbi et al. 1997a); HD $164615=$ V2118 Oph (Zerbi et al. 1997b); HR $8799=$ V342 Peg (Zerbi et al. 1999); HD 277 (Henry et al. 2001)].

Given the F1 V spectral type of HR 6844, its multiple periods in the range 0.6 to 1.5 days, the mean $B / V$ amplitude ratio of 1.39 , and the spectroscopic evidence of line profile variations, we concur with Handler \& Shobbrook (2002) that HR 6844 is a $\gamma$ Doradus variable.

\section{BASIC PROPERTIES OF HR 6844}

Although HR 6844 is a single-lined spectroscopic binary, it is possible to determine the basic properties of the system and its components with the help of several assumptions. The $V$ magnitude and parallax in the Hipparcos catalog (ESA 1997) plus the assumption of no interstellar extinction resulted in $M_{V}=2.64 \pm 0.11 \mathrm{mag}$. The $B-V$ color index of 0.312 mag from Hipparcos (ESA 1997) produced a bolometric correction and effective temperature from Table 3 of Flower (1996). Those two quantities in turn were used to compute the luminosity $L=6.78 \pm 0.71 L_{\odot}$ and the radius $R=1.70 \pm 0.11 R_{\odot}$ of the primary. These values are given in Table 5.

Since lines of the secondary are not seen in our redwavelength spectra, the secondary is $\gtrsim 2.5$ mag fainter than the primary (Stockton \& Fekel 1992). Thus, if the secondary is a main-sequence star, it has a spectral class of G5 or later (Gray 1992). The mass function of $0.000111 M_{\odot}$ indicates that the secondary mass is likely small, and the orbital inclination low. From its spectral type of F1 V (Gray \& Garrison 1989; Abt \& Morrell 1995) and Table B1 of Gray (1992), we adopt a mass of $1.51 M_{\odot}$ for the primary. Any mass for the secondary corresponding to a dwarf of $\mathrm{G}$ or $\mathrm{K}$ spectral class (Gray 1992) leads to an orbital inclination less than $10^{\circ}$, while an orbital inclination greater than $55^{\circ}$ results in a brown dwarf.

Matthews \& Mathieu (1992) examined 62 spectroscopic binaries with A-type primaries and periods less than 100 days. They concluded that all systems with orbital periods $\lesssim 3$ days have circular or nearly circular orbits. They also found that many binaries with periods in the range of 3-10 days have circular orbits. Thus, the adoption of a circular

TABLE 5

Fundamental Parameters of HR 6844

\begin{tabular}{|c|c|c|}
\hline Parameter & Value & Reference \\
\hline$V(\operatorname{mag}) \ldots \ldots$ & 6.62 & 1 \\
\hline$B-V(\operatorname{mag}) \ldots$ & 0.312 & 1 \\
\hline 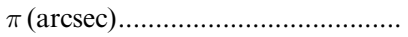 & $0.01598 \pm 0.00080$ & 1 \\
\hline Spectral type of component A ...... & $\mathrm{F} 1 \mathrm{~V}$ & 2 \\
\hline Spectral type of component B....... & M dwarf & 3 \\
\hline 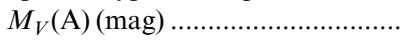 & $2.64 \pm 0.11$ & 3 \\
\hline 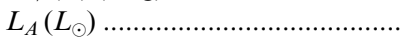 & $6.78 \pm 0.71$ & 3 \\
\hline 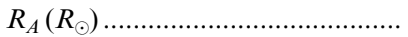 & $1.70 \pm 0.11$ & 3 \\
\hline
\end{tabular}

REFERENCES.-_(1) ESA 1997; (2) Gray \& Garrison 1989; (3) this paper. 
orbit for HR 6844 is quite consistent with these observational results.

Tidal forces act to circularize the orbits of binary stars and to synchronize the components' rotation periods with the orbital period. Zahn (1977) found that rotational synchronization should occur first and that systems consisting of stars with convective outer envelopes have shorter timescales for synchronization and circularization than binary components with radiative envelopes. With a spectral type of $\mathrm{F} 1 \mathrm{~V}$, the primary of HR 6844 has a radiative or, at best, a very thin convective outer atmosphere, while the secondary almost certainly has a significant convective atmosphere. If the primary is synchronously rotating, then the orbital period and its radius produce an equatorial rotational velocity of $19.1 \pm 1.3 \mathrm{~km} \mathrm{~s}^{-1}$. Fekel (1997) found $v \sin i=8.0 \pm 1.0 \mathrm{~km} \mathrm{~s}^{-1}$, so the rotational inclination is $25^{\circ}$. With the assumption that its rotational axis is aligned with the orbital axis plus the adopted primary mass, the mass function produces a mass of $0.16 M_{\odot}$ for the secondary. This corresponds to a mid or late $\mathrm{M}$ dwarf spectral type.

\section{DISCUSSION OF HR 6844}

Henry \& Fekel (2002) listed the 30 currently known $\gamma$ Doradus variables. Of that number, only five stars so far have been identified as spectroscopic binaries, and with the addition of HR 6844, three systems now have had their orbits determined. The other two stars are HD 62454 (Kaye et al. 1999b) and HD 209295 (Handler et al. 2002). Recently, Fekel et al. (2003) surveyed $34 \gamma$ Doradus candidates and found that at least $25 \%$ of that sample are spectroscopic binaries, and one of them, HD 221866, has just had its orbit determined (Kaye, Gray, \& Griffin 2003).

Of the four binaries with orbits, HD 209295 stands out, being quite unusual in several respects (Handler et al. 2002). The system has a short orbital period of 3.106 days, but the orbit, rather than being circular or nearly circular, has an eccentricity of 0.35 . In addition, Handler et al. (2002) concluded that the unseen secondary is likely a neutron star or white dwarf. From extensive photometric observations, they found that the F-type primary has both $\gamma$ Doradus and $\delta$ Scuti type pulsation, making it the first star to have both kinds of light variability. They detected nine frequencies in the $\gamma$ Doradus domain and one in the $\delta$ Scuti range. Five of the $\gamma$ Doradus frequencies are exact integer multiples of the orbital frequency, causing Handler et al. (2002) to suspect that those pulsation frequencies are excited by tidal interactions.

The orbital period and the spectral type of the primary in HR 6844 are quite similar to those of HD 209295, but the similarities end there, since the orbit of HR 6844 is circular and the secondary component is likely a very low mass, main-sequence star. Comparison of the orbital and light variation periods indicates that they are not related.

In $\gamma$ Doradus stars, line profile changes result in radial velocity variations. In 9 Aur (Krisciunas et al. 1995; Zerbi et al. 1997a), $\gamma$ Doradus (Balona et al. 1996), HD 164615 (Hatzes 1998), and HR 8330 (Aerts \& Kaye 2001), the periods of such velocity variations have been identified with periods of light variation. The amplitudes of the velocity variation range from $1.3 \mathrm{~km} \mathrm{~s}^{-1}$ in 9 Aur to $\sim 4 \mathrm{~km} \mathrm{~s}^{-1}$ in HD 164615. The radial velocity amplitude of $\sim 3 \mathrm{~km} \mathrm{~s}^{-1}$ found for HR 6844 falls within this range.

\section{RESULTS FOR $73 \mathrm{Oph}(=\mathrm{HR} 6795=\mathrm{HD}$ $166233=$ ADS 11111AB)}

Our F2 V check star 73 Oph is a close visual binary with an orbital period of $294 \mathrm{yr}$ (Söderhjelm 1999). The Hipparcos catalog lists the secondary as 1.34 mag fainter than the primary and 0".43 distant (ESA 1997). From 23 observations over $7 \mathrm{hr}$, Percy (1973) suspected that the star is a lowamplitude photometric variable. On that basis, it was assigned suspected variable star number NSV 10287 (Kholopov 1982).

The $K-C$ data sets in $B$ and $V$ were modified to remove most of the monitoring night data, as described in $\S 3.2$ above. The least-squares spectra resulting from our analysis of the $B$ data are shown in Figure 7. The analysis was done over the frequency range of $0.01-30.0 \mathrm{day}^{-1}$, but the figure plots only the lower frequencies where variability was observed. A single frequency at $1.62762 \mathrm{day}^{-1}$ is evident, corresponding to a period of 0.61439 days. Essentially the same period is found in the $V$ data set. The observed peakto-peak amplitudes are 8.6 and $6.2 \mathrm{mmag}$ in $B$ and $V$, respectively. The period is close to the third period found in HR 6844, but, given its small uncertainty and very different amplitude from the similar period in HR 6844, it is definitely an independent period. Thus, we do not find any common frequencies in the $V-C$ and $K-C$ data sets, and we can uniquely assign the three frequencies in the $V-C$ data set to HR 6844 and the single frequency in the $K-C$ data set to 73 $\mathrm{Oph}$, i.e., none of the detected frequencies arises from the comparison star.

The $B$ observations, phased with the single frequency and time of minimum from Table 4, are shown in Figure 8. Like HR 6844, the light curve is sinusoidal but with no evidence of excess scatter around maximum brightness. The observed $B / V$ amplitude ratio of 1.39 suggests that the photometric variability arises from pulsations and not from photospheric spots or the ellipticity effect (if one of the two visual binary components is an undetected, short-period spectroscopic binary), which would result in amplitude ratios of $\sim 1.1$ and $\sim 1.0$, respectively (Henry et al. 2000).

Although we have no spectra of $73 \mathrm{Oph}$, a few radial velocities are available in the literature. Six observations from 1921 to 1923 at Mount Wilson Observatory have a mean of $-21 \mathrm{~km} \mathrm{~s}^{-1}$ and a spread $(\mathrm{rms})$ of $12 \mathrm{~km} \mathrm{~s}^{-1}$

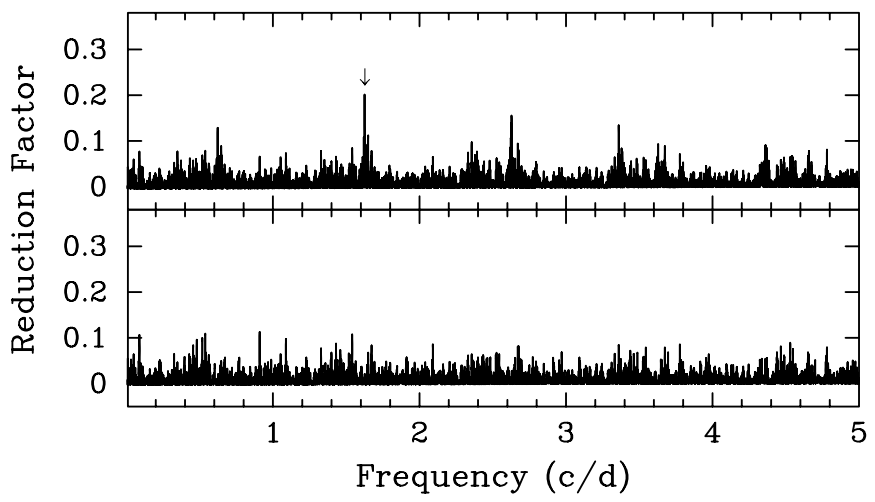

FIG. 7.-Least-squares spectra of our Johnson $B$ check star data for $73 \mathrm{Oph}$. The arrow in the top panel indicates the single detected frequency at $1.62762 \mathrm{day}^{-1}$. The bottom panel shows the least-squares spectrum with the 1.62762 day $^{-1}$ frequency fixed. The same frequency was confirmed in the Johnson $V$ data set. 


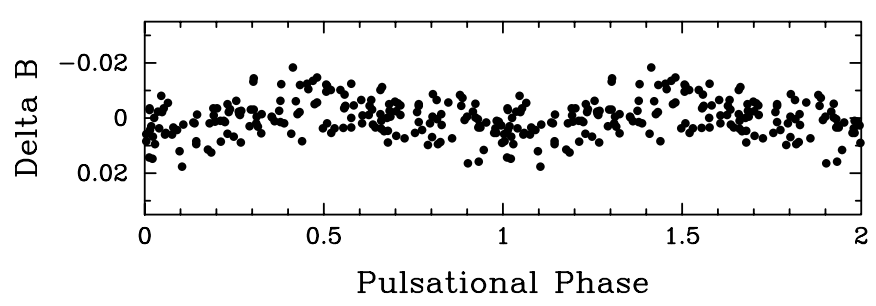

FIG. 8.- Johnson $B$ photometric data for $73 \mathrm{Oph}$, phased with the single frequency of $1.62762 \mathrm{day}^{-1}$ and time of minimum from Table 3 .

(Wilson \& Joy 1950; Abt 1973). Another six observations in 1919 and 1920 by Plaskett et al. (1921) at Dominion Astrophysical Observatory have a mean of $-14.6 \mathrm{~km} \mathrm{~s}^{-1}$ and an rms of only $3.8 \mathrm{~km} \mathrm{~s}^{-1}$. Thus, the lack of high-amplitude, short-period, radial velocity variations makes it unlikely that the 0.61 day photometric period in $73 \mathrm{Oph}$ is due to ellipticity in one of the visual binary components.

Fabricius \& Makarov (2000) derived Tycho $B_{T}$ and $V_{T}$ magnitudes of the individual components of $73 \mathrm{Oph}$. We used these magnitudes, along with the transformation equations from Volume 1 of the Hipparcos catalog (ESA 1997), to compute Johnson $V$ magnitudes and $B-V$ color indices. For the primary, we find $V=6.03$ and $B-V=0.32$; for the secondary, $V=7.96$ and $B-V=0.60$. Combined with the Hipparocs parallax of 0..01962, these results imply F0 V and G1 V spectral types for the primary and secondary, respectively. This places the primary right in the heart of the $\gamma$ Doradus instability region of the H-R diagram (Henry \& Fekel 2002). We assume the photometric variability arises from the $\mathrm{F} 0 \mathrm{~V}$ primary since it is several times brighter than the G1 V secondary and possible starspot variability in the secondary is very unlikely to produce the coherent photometric variability observed over 1240 cycles. Therefore, we can correct the photometric amplitudes of the primary for dilution by the secondary, and we find them to be 9.7 and
TABLE 6

Fundamental Parameters of the Check Star 73 Oph

\begin{tabular}{|c|c|c|}
\hline Parameter & Value & Reference \\
\hline 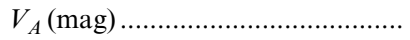 & 6.03 & 1 \\
\hline 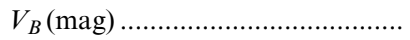 & 7.96 & 1 \\
\hline 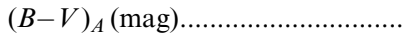 & 0.318 & 1 \\
\hline 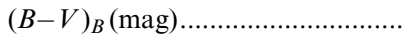 & 0.599 & 1 \\
\hline 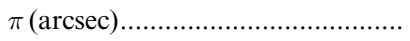 & $0.01962 \pm 0.00122$ & 2 \\
\hline Spectral type of component A ....... & $\mathrm{F} 0 \mathrm{~V}$ & 1 \\
\hline Spectral type of component B....... & G1 V & 1 \\
\hline 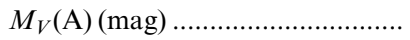 & $2.49 \pm 0.14$ & 1 \\
\hline$M_{V}(\mathrm{~B})(\mathrm{mag}) \ldots \ldots \ldots \ldots \ldots \ldots \ldots \ldots \ldots \ldots$ & $4.43 \pm 0.15$ & 1 \\
\hline$L_{A}\left(L_{\odot}\right) \ldots \ldots \ldots \ldots \ldots \ldots$ & $7.78 \pm 1.00$ & 1 \\
\hline$L_{B}\left(L_{\odot}\right) \ldots \ldots \ldots \ldots \ldots \ldots \ldots \ldots \ldots \ldots \ldots \ldots \ldots \ldots \ldots \ldots \ldots \ldots$ & $1.42 \pm 0.18$ & 1 \\
\hline 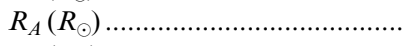 & $1.83 \pm 0.14$ & 1 \\
\hline 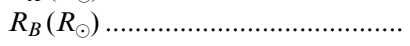 & $1.15 \pm 0.08$ & 1 \\
\hline
\end{tabular}

REFERENCES.-(1) This paper; (2) ESA 1997.

$7.2 \mathrm{mmag}$ in $B$ and $V$, respectively. This gives a corrected $B / V$ amplitude ratio of 1.34 , very similar to the amplitude ratio in HR 6844 and other $\gamma$ Doradus variables (Henry \& Fekel 2002). The properties of both components are listed in Table 6.

With a spectral type of F0 V, a period of 0.61 days, an amplitude ratio of 1.34, and a lack of high-amplitude, shortperiod, radial velocity variations, $73 \mathrm{Oph}$ is almost certainly a new $\gamma$ Doradus star.

We are grateful to Lou Boyd of Fairborn Observatory for supporting the automated telescopes. We thank David Barlow for computing a solution of the radial velocities. The research at Tennessee State University is supported in part by NASA grants NCC 5-511 and NCC 5-96 and NSF grant HRD-9706268.
Abt, H. A. 1973, ApJS, 26, 365

Abt, H. A., \& Morrell, N. I. 1995, ApJS, 99, 135

Aerts, C., Eyer, L., \& Kestens, E. 1998, A\&A, 337, 790

Aerts, C., \& Kaye, A. B. 2001, ApJ, 553, 814

Balona, L. A., et al. 1996, MNRAS, 281, 1315

Barker, E. S., Evans, D. S., \& Laing, J. D. 1967, R. Obs. Bull., 130, 355

Batten, A. H., Fletcher, J. M., \& MacCarthy, D. G. 1989, Publ. Dom.

Astrophys. Obs., 17, 1

Cowley, A., \& Fraquelli, D. 1974, PASP, 86, 70

Danziger, I. J., \& Faber, S. M. 1972, A\&A, 18, 428

ESA. 1997, The Hipparcos and Tycho Catalogues (ESA SP-1200) (Noorwijk: ESA)

Fabricius, C., \& Makarov, V. V. 2000, A\&A, 356, 141

Fekel, F. C. 1997, PASP, 109, 514

Fekel, F. C., Henry, G. W., Eaton, J. A., Sperauskas, J., \& Hall, D. S. 2002, AJ, 124, 1064

Fekel, F. C., Warner, P. B., \& Kaye, A. B. 2003, AJ, in press

Fitzpatrick, M. J. 1993, in ASP Conf. Ser. 52, Astronomical Data Analysis

Software and Systems II, ed. R. J. Hanisch, R. V. J. Brissenden, \& J. Barnes (San Francisco: ASP), 472

Flower, P. J. 1996, ApJ, 469, 355

Gray, D. F. 1992, The Observation and Analysis of Stellar Photospheres (2d ed.; Cambridge: Cambridge Univ. Press)

Gray, R. O., \& Garrison, R. F. 1989, ApJS, 69, 301

Guzik, J. A., Kaye, A. B., Bradley, P. A., Cox, A. N., \& Neuforge, C. 2000, ApJ, 542, L57

Handler, G. 1999, MNRAS, 309, L19

Handler, G., et al. 2002, MNRAS, 333, 262

Handler, G., \& Shobbrook, R. R. 2002, MNRAS, 333, 251

Hatzes, A. P. 1998, MNRAS, 299, 403

Henry, G. W., \& Fekel, F. C. 2002, PASP, 114, 988

Henry, G. W., Fekel, F. C., Henry, S. M., \& Hall, D. S. 2000, ApJS, 130, 201

Henry, G. W., Fekel, F. C., Kaye, A. B., \& Kaul, A. 2001, AJ, 122, 3383

\section{REFERENCES}

Kaye, A. B., Gray, R. O., \& Griffin, R. F. 2003, PASP, submitted

Kaye, A. B., Handler, G., Krisciunas, K., Poretti, E., \& Zerbi, F. M. 1999a, PASP, 111,840

Kaye, A. B., Henry, G. W., Fekel, F. C., \& Hall, D. S. 1999b, MNRAS, 308, 1081

Krisciunas, K., Griffin, R. F., Guinan, E. F., Luedeke, K. D., \& McCook, G. P. 1995, MNRAS, 273, 662

Kukarkin et al. 1982, New Catalogue of Suspected Variable Stars (Moscow: Nauka)

Lucy, L. B., \& Sweeney, M. A. 1971, AJ, 76, 544

Matthews, L. D., \& Mathieu, R. D. 1992, in ASP Conf. Ser. 32, Complimentary Approaches to Double and Multiple Star Research, ed. H. A McAlister \& W. I. Hartkopf (IAU Colloq. 135) (San Francisco: ASP), 244

Paunzen, E. \& Maitzen, H. M. 1998, A\&AS, 133, 1

Percy, J. R. 1973, JRASC, 67, 139

Plaskett, J. S., Harper, W. E., Young, R. K., \& Plaskett, H. H. 1921, Publ. Dominion Astrophy. Obs., 2, 1

Scarfe, C. D., Batten, A. H., \& Fletcher, J. M. 1990, Publ. Dominion Astrophy. Obs., 18, 21

Söderhjelm, S. 1999, A\&A, 341, 121

Stockton, R. A., \& Fekel, F. C. 1992, MNRAS, 256, 575

Strassmeier, K. G., Washuettl, A., Granzer, T., Scheck, M., \& Weber, M. 2000, A\&AS, 142, 275

Szeidl, B. 1976, in IAU Colloq. 29, Multiple Periodic Variable Stars, ed.

W. S. Fitch (Dordrecht: Reidel), 133

Vanicek, P. 1971, Ap\&SS, 12, 10

Wilson, R. E., \& Joy, A. H. 1950, ApJ, 111, 221

Wolfe, R. H., Horak, H. G., \& Storer, N. W. 1967, in Modern Astrophysics, ed. M. Hack (New York: Gordon \& Breach), 251

Zahn, J.-P. 1977, A\&A, 57, 383

Zerbi, F. M., et al. 1997a, MNRAS, 290, 401

. 1997b, MNRAS, 292, 43 1999, MNRAS, 303, 275 